\title{
Damping of waves of agitation in starling flocks
}

\author{
C. K. Hemelrijk ${ }^{1}$ A. Costanzo ${ }^{1} \cdot$ H. Hildenbrandt ${ }^{1} \cdot$ C. Carere $^{2}$
}

Received: 8 February 2019 / Revised: 17 July 2019 / Accepted: 29 July 2019 / Published online: 15 August 2019

(C) The Author(s) 2019

\begin{abstract}
When a predator attacks a flock of starlings (Sturnus vulgaris), involving thousands of individuals, a typical collective escape response is the so-called agitation wave, consisting of one or more dark bands (pulses) propagating through the flock and moving away from the predator (usually a Peregrine falcon, Falco peregrinus). The mechanism underlying this collective behavior remains debated. A theoretical study has suggested that the individual motion underlying a pulse could be a skitter (in the form of a zigzag), that is copied by nearby neighbors, and causes us to temporarily observe a larger surface of the wing because the bird is banking during turning while zigzagging. It is not known, however, whether pulses during a wave event weaken over time. This is of interest, because whereas during the usual turning by an undisturbed flock the motion is copied completely without weakening, we may expect that pulses dampen during a wave event because individuals that are further away from a predator react less because of reduced fear. In the present paper, we show in empirical data that pulses during a wave event weaken over time. Our computational model, StarDisplay, reveals that this is most likely a consequence of a reduction of the maximum banking angle during the zigzag escape maneuver rather than by a reduced tendency to copy this maneuver with time. The response seems adaptive because of lowered danger at a larger distance to the location of attack.
\end{abstract}

\section{Significance statement}

Huge flocks of starlings display amazing patterns of collective escape when attacked by an avian predator, such as a Peregrine falcon. One of them is the "agitation wave" in which dark bands move away from the predator. Dark bands arise probably from the temporarily larger wing area, which is observed when birds perform a skitter escape motion (zigzag) while temporarily banking sideward. Whereas during regular flock turns birds copy each other's motion completely, it is unknown whether this happens during agitation waves, because individuals further away from the attack may be less frightened. Studying this both empirically at the group level only and in a computational model at both the level of the individual and the group, we show that pulses of waves fade out with time and that this is probably due to a reduced maximum banking angle during the zigzag maneuver rather than a lower tendency of copying. This seems an adaptive response.

Keywords Flocking $\cdot$ Starling $\cdot$ Predation $\cdot$ Agitation wave $\cdot$ Collective escape $\cdot$ Zigzag

Communicated by P. A. Bednekoff

Electronic supplementary material The online version of this article (https://doi.org/10.1007/s00265-019-2734-4) contains supplementary material, which is available to authorized users.

C. K. Hemelrijk

c.k.hemelrijk@rug.nl

1 Theoretical Research in Evolutionary Life Sciences, TRÊS, Faculty of Science and Engineering, FSE, Groningen Institute for Evolutionary Life Sciences, Centre for Life Sciences, University of Groningen, Nijenborgh 7, 9747 AG Groningen, The Netherlands

2 Department of Ecological and Biological Sciences, University of Tuscia, viale dell'Università s.n.c., 01100 Viterbo, Italy

\section{Introduction}

The study of collective motion of social animals, including humans, is an interdisciplinary field at the frontiers of science, which has greatly benefitted from combining empirical and modeling approaches (Camazine et al. 2003; Grimm et al. 2005; Sumpter et al. 2012; Ouellette 2019). When starlings swirl above their roosting site in huge flocks of thousands of individuals, they perform even without a predator attacking them complex maneuvers involving sudden collective turns that change the shape and density of flocks remarkably. When the flocks are attacked by a predator, they display patterns of collective escape that are even more complex (Feare 1984; Ballerini et al. 2008a; Carere et al. 2009; Goodenough et al. 2017; Storms et al. 2019). 
One of the most common patterns of collective escape is the so-called "wave of agitation," where one or more dark bands (here referred to as pulses) of an approximately fixed width originate close to the attacking predator and travel within a second over the flock away from it (Procaccini et al. 2011; Storms et al. 2019); thus, the wave is transferring information rapidly across many individuals (Gerlotto et al. 2006; Procaccini et al. 2011). The function of this wave may be that it confuses the predator in whom to attack (Procaccini et al. 2011). How individuals behave and transfer information to produce such pulses is still unknown. Information transmission in flocks of starlings has been studied empirically so far only during collective turning (Attanasi et al. 2014; Cavagna et al. 2015). Based on detailed empirical data recorded with three cameras, it has been shown that once an individual had started a turn, others followed at approximately constant intervals, with an identical turning motion (Attanasi et al. 2014). Whether during an agitation wave individuals copy and repeat escape maneuvers identically or weaken the maneuver over time, so that the pulse would fade out, remains unknown. This question is of interest, because in our recent analysis of reactions of a flock to a predator, we quantitatively showed that the patterns of collective response of starlings are fine tuned to the characteristics of hunting by a falcon (such as its speed and direction from which it attacks; Storms et al. 2019). Similarly, weakening of a wave over time may be adaptive and reducing fear and responsiveness to a predator when it is further away is in line with theoretical predictions (Broom and Ruxton 2005).

In the present study, we investigate agitation waves both empirically and theoretically. Empirically, we analyze agitation waves elicited by avian predators (Peregrine falcon) and video-recorded above urban roosts in Rome, Italy. Empirical analysis can be done only at the group level because of the great distance between observers and the flocks. Theoretically, we use our earlier computational model, StarDisplay, in which flocks resemble empirical data in many ways (Hemelrijk and Hildenbrandt 2011; Hemelrijk and Hildenbrandt 2015a, b; Hemelrijk et al. 2015). In it, we showed that individual motion underlying the wave of agitation was a skitter motion (zigzagging) resulting in an "orientation wave" rather than a motion of fleeing inward into the flock causing a density wave (in which individuals temporarily cluster closer together) (Hemelrijk et al. 2015). The change of orientation of the wing towards the observer during the simultaneous skitter motion of several individuals caused the dark bands. We continue to investigate the skitter maneuver by studying what happens at the individual level in more detail, and producing in the model agitation waves that are either damped in their amplitude of regular variation of darkness or not.

We posed the following questions: (1) Does the intensity of darkness of an agitation wave decrease with time as it progresses through the flock? (2) How does the regular variation of darkness in the model compare to that in real flocks in terms of decrease of darkness of pulses, if we assume in the model (a) a complete tendency to repeat the "zigzag" maneuver, (b) a reduced tendency to repeat it, and (c) a complete tendency to repeat it, but by banking to a smaller maximum angle?

\section{Methods}

We investigated whether pulses of agitation waves in empirical data of starling flocks under predation threat collected in Rome, Italy (Procaccini et al. 2011) fade out and compared this with pulses, which were generated in a computational model of starling flocks, StarDisplay (Hemelrijk et al. 2015).

\section{Empirical footage and its analysis}

We collected data on 16 wave events including a total of 44 pulses from the footage of 5 starling flocks recorded in Rome across two winter seasons as described in a previous study (Procaccini et al. 2011). We distinguish between wave events and pulses following the definition by Procaccini and coauthors (Procaccini et al. 2011), whereby a wave event comprises a single pulse of darkness (Figs. 1 and 2) or a series of pulses (Fig. 2b) that travel in a given direction through the flock and a wave event is separated from the next one by an interval of $10 \mathrm{~s}$. We identify a pulse in the plots of regular variation of luminance applying the following criteria: (a) there should be a decrease of luminance from a local maximum until a local minimum and (b) the regular variation of luminance of each specific pulse should be visible at its start location (red line in Fig. 2b) before being visible at its end location (blue Figs. $2 \mathrm{~b}$ and S1; Table S1). The wave ends by the last oscillation, thus where there is a last local maximum followed by a local minimum in both, the location at the beginning and end.

We selected those videos with agitation waves in which flock and falcon were clearly visible, the shape of the flock was relatively undisturbed, and not deforming too much and in which the predator attacked at a single location in the flock without changing attack location during the wave event.

To determine whether the darkness of pulses decreases with time, we measure per wave event and per pulse the difference in amplitude of the regular variation of luminance at a location (so-called region of interest) close to where the wave starts (starting location) and at a location where the wave approximately ends (end location) (Figs. 1 and 2). Fading out of waves is indicated by a statistically positive difference over all waves between the amplitude at beginning minus that at its end for each wave event or pulse. For some wave events, we average values of maximally 3 measurements at the beginning and at the end of the pulse. To measure the amplitude at a specific location of the flock (namely at the start location and end location), 
Fig. 1 An example of measuring the regular variation of luminance $\mathbf{a}$ in empirical data and $\mathbf{b}$ in computational model between $t=$ $0 \mathrm{~s}$ (above) and $1 \mathrm{~s}$ (below) in two regions of interest (indicated as two squares) in a starling flock at a start location (square indicated by broken line) and end location (square indicated with continuous line). The dotted lines indicate the period of $1 \mathrm{~s}$ over which frames are interpolated during the motion of the region of interest. The arrow shows the direction of motion of the pulse over the flock

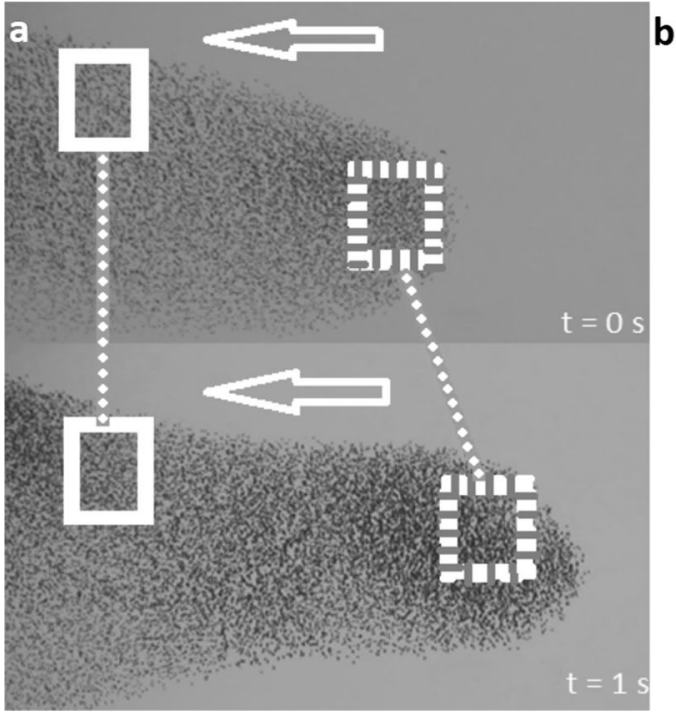

we need to follow the location of this region over time (Fig. 1). We designed a computer program for linearly interpolating these two positions of a specific region of interest over time. The amplitude of the luminance $L(t)$ at a specific location is normalized by the average luminance of the background and computed per wave event and per pulse as $\max [L(t)]-\min [L(t)] / \operatorname{avg}\left[L \_b g(t)\right]$, whereby maximum and minimum are measured either over the complete wave or per pulse respectively. The average value of the normalized luminance in the region of interest is the value for Y from the YUV color space divided by 255, the maximum value of the $\mathrm{Y}$ channel. Here, $\mathrm{Y}$ stands for the luminance and $\mathrm{U}$ and $\mathrm{V}$ for the range of blue-yellow and redcyan colors respectively. A normalized luminance of 1

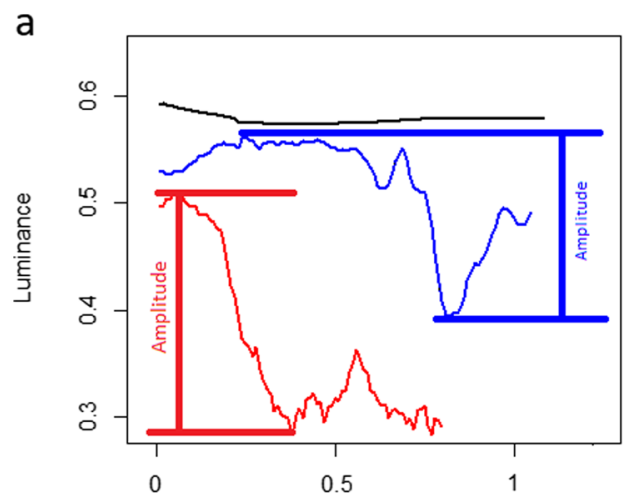

$\mathrm{t}(\mathrm{s})$

Fig. 2 Measurement of amplitude of regular variation of luminance at two levels, more coarsely, a per wave event and more refined, b per pulse. a The amplitude of the luminance per wave event is measured over the whole wave event at the location at the beginning (red), namely as indicated in the discretized square in Fig. 1 and at the location at the end of the wave (blue) (see continued square in Fig. 1), and in the background (black line) over time (in sec). b The amplitude is measured per pulse (subsequent pulses are indicated by increasing numbers) at both locations, beginning and end (see Fig. 1). represents a plain, white area and 0 a plain, black area. We calculated amplitudes at the level of a wave event and at the level of pulses (as defined above) as follows (Fig. 2a, b). At the level of the wave event, we measured the maximum amplitude at each of the two locations by subtracting the minimum of luminance from the maximum, at the location at the beginning and at the end (Fig. 2a). At the level of the pulse, we measured the amplitude per pulse at both locations (Figs. $2 \mathrm{~b}$ and $\mathrm{S} 1$ ).

The amplitudes of regular variation of luminance were not normally distributed (Figs. 3a, b and S3) and sample size was small, so we used nonparametric tests only, namely the Wilcoxon matched-pairs signed-ranks test and Kendall rank correlations.

b

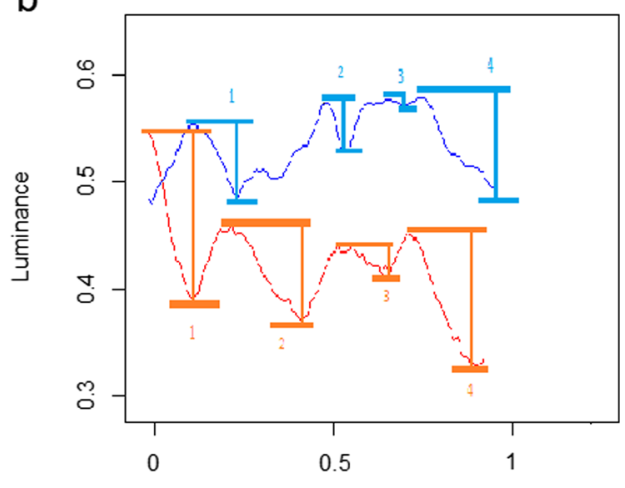

$t(s)$

Amplitudes of the beginning (in red) and end locations (blue) of each pulse are indicated and pulses are numbered consecutively (for pulses measured in all wave events; see Fig. S1). The amplitude per wave event and per pulse is measured in both cases as $\max [L(t)]-\min [L(t)]$ where the maximum and minimum are calculated per wave or pulse respectively. Controlled for the average of the background over time (indicated as $(\operatorname{avg}[\operatorname{bg}(\mathrm{t})])$, it is Amplitude $=\frac{\max [L(t)]-\min [L(t)]}{\operatorname{avg}[\operatorname{bg}(t)]}$ 

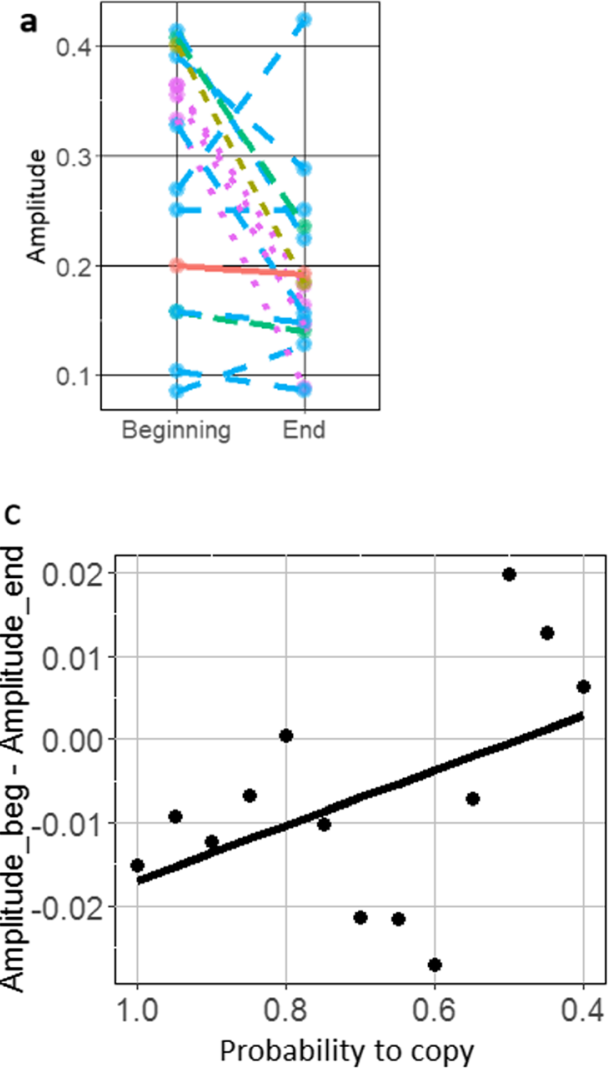

Fig. 3 Amplitudes of the modulating luminance normalized by the average luminance of the background at the location at the beginning and end of a 16 wave events and b 44 pulses in 16 wave events in 5 real flocks (see supplementary Fig. S7). c Difference in amplitude of the modulating luminance in the model at the location at the beginning and end of a wave for a decreasing probability to copy the escape maneuver. $\mathbf{d}$ Same difference in amplitude in the model against a decreasing angle of banking during the escape maneuver of zigzagging. The differences in amplitudes of luminance between the locations at the beginning and end were mostly not distributed normally: Shapiro-Wilk normality test in empirical data: $n=16$ wave events, $W=0.89, p=0.07$ two-tailed; $n=$ 44 pulses, $W=0.96, p=0.10$ two-tailed; modeling results of probability of copying: $n=13, W=0.96, p=0.70$ two-tailed and thus followed up by

\section{Simulation experiments in the model StarDisplay}

We use our model of collective motion of starling flocks, StarDisplay (Hildenbrandt et al. 2010; Hemelrijk and Hildenbrandt 2011, 2015a, b; Hemelrijk et al. 2015), since its flocks resemble real flocks in many traits, including its agitation waves (Hemelrijk et al. 2015, see also "Introduction"). This model, parametrized to empirical data of real starlings (Table S2), is based on coordination among birds, in which individuals are attracted to others, align with them, avoid collisions with them, and fly following a simplified version of flying behavior based on fixed wing aerodynamics (Hildenbrandt et al. 2010; Hemelrijk and Hildenbrandt 2011, 2012, 2015a, b; Hemelrijk et al. 2015). Following empirical findings, in the model, individuals are attracted to

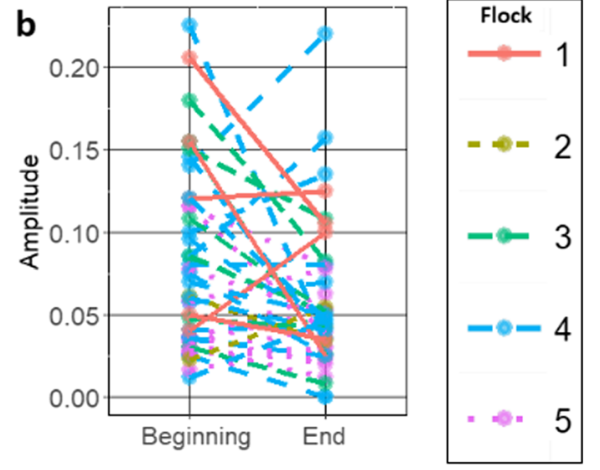

d

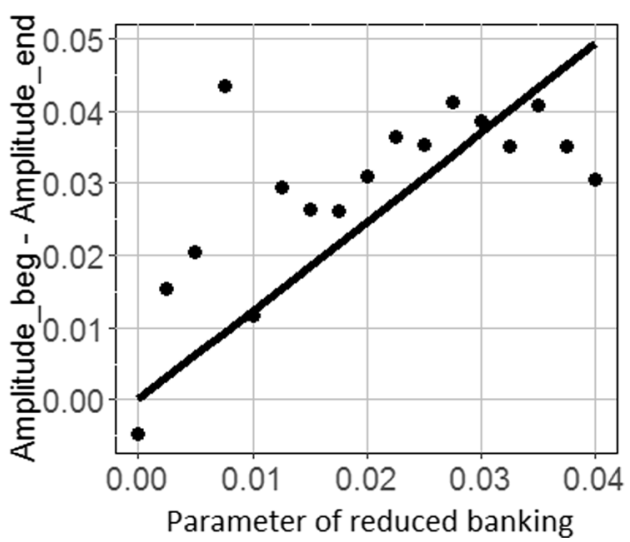

nonparametric tests; apart from the differences for reduced depth of banking: $n=17, W=0.88, p=0.04$ two-tailed, which were normally distributed and subsequently tested parametrically. $\mathbf{a}, \mathbf{b}$ The amplitude of luminance is on average larger at the location at the beginning than at its end: Wilcoxon matched-pairs signed-ranks test: at level of wave events: $n=$ $16, W=108, p=0.007$ two-tailed; at level of pulses: $n=44, W=677.5$, $p=0.014$ two-tailed. $\mathbf{c}$ Decreasing the tendency to copy in the model has a non-significant effect (Kendall rank correlation: $n=13$, tau $=-0.23$, $p=0.31$ two-tailed). $\mathbf{d}$ If individuals copy the zigzag with a decreasing maximum banking angle, the wave fades out (Pearson product-moment correlation: $d f=15, r=0.67, p=0.0003$ two-tailed). For the decrease in angle of banking, see Fig. S2, and for normalized luminance at both locations over time with decreasing banking angles, see Fig. S8

staying above the area for sleeping (the roosting site) and coordinate with only their 7 closest neighbors (Ballerini et al. 2008b). For more detail on the basic model, see supplementary material. Here, we focus on details of modeling the agitation wave in flocks of 2000 individuals. Birds are represented as black delta-shaped objects and waves are initiated by a single bird at the border of the flock that supposedly detects a predator. The escape maneuver is a skitter motion, in the form of a "zigzag" (Table S3). Here, a bird follows a bend path in which it rolls (banks) temporarily in one direction and rolls back again. In the original model (Hemelrijk et al. 2015), the neighbors nearby copy this motion exactly, which causes dark bands to propagate over the flock. In the present study, we have adjusted the copying of the escape maneuver in two ways: (1) we reduce the chance that neighbors copy it, and 
(2) we make the neighbors roll less deep (but as long) at each event of copying the escape maneuver. We have studied the effect of various parameters in a single wave event where individuals were copying (or not) the escape maneuver from the 7 closest neighbors. The parameter range of the probability of copying was limited between 1.00 and 0.4 with decrements of 0.5 , thus, $1.00,0.95,0.9$, etcetera, in order to keep individuals together in the flock despite their difference in motion. The parameters of the reduction of the maximum angle of tilting with subsequent events of copying ranged from 0 to 0.04 , with increments of 0.0025 , meaning that the maximum angle of tilting was reduced by that fraction $(0 \%, 0.25 \%$, $0.5 \%, 0.75 \%, 1 \%$, etcetera). Here, we reduced the angle of banking (called CopyDecay in Fig. S6) by a fraction while keeping the same time interval of banking.

We generate two-dimensional images of the flock on a white background by making the observer (or camera) move parallel to the flock in the same direction as the flock and pointing to the center of gravity of the flock (at an almost fixed angle) so that it perceives maximal changes in wing surface. The amplitude of regular variations of luminance of the flock is measured in the same way as in the empirical footage.

\section{Results}

In videos of real flocks, we analyzed 16 wave events that comprised one to 6 pulses of darkness traveling through the flock (a total of 44 pulses; Table S1).

The results show that the amplitude of the regular variation of luminance is on average larger at the location at the beginning than the location at its end (Fig. 3a, b; for both methods, see Fig. 2a, b; for the difference in amplitude at location at beginning and end of a pulse for each pulse per wave, see Fig. S7 and for separate pulses, see Fig. S1). This means that empirically a pulse fades out.

In our computational model, StarDisplay, we represent the weakening of pulses in two ways by decreasing (a) the tendency to copy over time, and (b) the maximum angle of tilting during a skitter-like escape maneuver (the zigzag). In the model, we see that decreasing the tendency to copy has no significant effect on the amplitude at the end position (Fig. 3c). Yet, if individuals copy the zigzag with a decreasing maximum banking angle, the pulse in our model weakens like in empirical data (Figs. 3d and S8).

\section{Discussion}

The empirical data from attacks by falcons on real flocks of starlings show that agitation waves and their pulses significantly decrease in intensity and thereby, transmission of information about a threat decreases with time. To produce a decay in a wave in our computational model, StarDisplay, individuals need to repeat the zigzag-like escape maneuver while reducing its prominence over time, meaning that individuals are banking to a maximum angle that is smaller with each subsequent event of copying. Decay in intensity of the pulses happens neither if individuals copy the escape maneuver completely from their nearby neighbors nor if they reduce their tendency to copy it. Thus, our study suggests that in real flocks, waves and pulses fade out due to decreasing the maximum exposure of the surface of the wings to the observer during copying the zigzag escape maneuvers over time.

This extends our former results when using StarDisplay for studying what underlies agitation waves (Hemelrijk et al. 2015). Here, we suggested that waves either reflected a change of density of individuals in the flock (Axelsen et al. 2001; Procaccini et al. 2011) or their orientation (Buchanan et al. 1988; Potts 1984) or both (Hemelrijk et al. 2015). We showed that only when the wave through the flock comprised a zigzag escape maneuver, our visual system observed indeed a dark band moving along the flock, not when the escape maneuver involved individuals fleeing fast forward into the flock. The orientation wave (skitter wave by zigzagging) in the model moved away from the predator at a speed similar to that in empirical data of the agitation wave of real flocks (Procaccini et al. 2011). The information transmission during copying in the model was complete, and therefore, individuals were always repeating a zigzag when at least one of their influential neighbors performed it and were repeating it in exactly the same way as they observed it. Our present findings show that our earlier model of agitation waves differs from empirical data in its lack of decay in amplitude of regular variation of luminance with time (Hemelrijk et al. 2015).

Our findings seem to warrant the use of the model StarDisplay for testing different hypotheses, because it adds an extra pattern (of damping of waves) to several other emergent properties of modeled flocks that resemble empirical data outside the context of predation. These are the flocks' relative proportions of height, width, and length; their similar density at front and back; collective turning over equal radii, which produces changes of shape during turning; the scale free correlation between collective heading and flock size, and the degree of diffusion in the flock (Hemelrijk and Hildenbrandt 2008, 2011, 2012, 2015a, b; Hildenbrandt et al. 2010; Hemelrijk et al. 2015).

As a possible limitation of our study, we acknowledge that our empirical data are based on events in which flocks reacted to a single predator, even though waves were sometimes generated by more than one predator. We omitted these few episodes because they were too complex for our analysis. Besides, we also ignored waves generated by a non-predator passing by (such as a gull), because these waves are weaker and die out sooner. Although we expect our explanation to hold here also, this needs further study. 
A decreasing maximum banking angle when copying a maneuver of turning while zigzagging differs from what is empirically observed in starling flocks during "regular" collective turning in the absence of a negative stimulus such as a predator. During regular turning when the group is undisturbed, individuals appear all to be turning over the same angle (Attanasi et al. 2014). The fading out of the turning in the zigzag-skitter motion with each subsequent event of copying may be a consequence of decreasing fearfulness of birds the larger their distance to the predator. Such decrease in responsiveness could be adaptive in terms of energy expenditure, because at a larger distance from the predator, the chance to be caught is smaller (Broom and Ruxton 2005). Individual starlings are sensitive to subtle cues of predation risk and rapidly resume normal behavior, e.g., foraging, if these cues are gone (Carter et al. 2008).

Future studies based on filming at closer proximity (possibly with the help of drones) are needed to explore in detail how fading out of skitter motions during escape maneuvers compare to the individual behavior during undisturbed turns of the flock.

Acknowledgments The video material for this project was gathered during a NEST program (STREP project "StarFlag" no. 12682) aimed at studying animal group movements where $\mathrm{CKH}$ and $\mathrm{HH}$ coordinated the unit on flocking models, and Enrico Alleva and Daniela Santucci, the unit collecting field data. Field video recording was carried out by $\mathrm{CC}$ and Francesca Zoratto. We thank the Self-Organization Lab for helpful discussions, particularly Rolf Storms and Kim Kiekebos for initial work on the analyses.

Author contributions $\mathrm{CKH}$ and HH conceived the idea of this paper; $\mathrm{CKH}$ wrote the main part of the manuscript and contributed to the statistical analysis. HH supervised the analysis of empirical data, did the computational modeling and the analysis of the modeling data, made some Figs, and contributed to the writing. CA analyzed the empirical data, made most of the Figs, and wrote part of the methods. CC collected the data, contributed to discussions, and participated in the writing.

Funding information This work is financed by the Netherlands Organisation for Scientific Research (NWO), the Open Technology Programme (OTP) Preventing bird strikes: Developing RoboFalcons to deter bird flocks, project number 14723, awarded to CKH. Cooperation with CC was funded by two visitor grants from NWO (040.11.468 and 040.11.573/1798) to CKH.

Data availability The datasets generated and/or analyzed during the current study are available in the Figshare https://doi.org/10.6084/m9. figshare.8104724.

\section{Compliance with ethical standards}

Conflict of interests The authors declare that they have no conflict of interest.

Ethical approval This study is purely observational and did not involve captive animals or experimental manipulations, only analyses of video material. The video recordings of the flocks and falcons were taken from hundreds of meters distance in an urban environment and did not cause any disturbance to the birds.

Open Access This article is distributed under the terms of the Creative Commons Attribution 4.0 International License (http:// creativecommons.org/licenses/by/4.0/), which permits unrestricted use, distribution, and reproduction in any medium, provided you give appropriate credit to the original author(s) and the source, provide a link to the Creative Commons license, and indicate if changes were made.

\section{References}

Attanasi A, Cavagna A, Del Castello L et al (2014) Information transfer and behavioural inertia in starling flocks. Nat Phys 10:691-696

Axelsen BE, Anker-Nilssen T, Fossum P, Kvamme C, Nottestad L (2001) Pretty patterns but a simple strategy: predator-prey interactions between juvenile herring and Atlantic puffins observed with multibeam sonar. Can J Zool 79:1586-1596

Ballerini M, Cabibbo N, Candelier R, Cavagna A, Cisbani E, Giardina I, Orlandi A, Parisi G, Procaccini A, Viale M, Zdravkovic V (2008a) Empirical investigation of starling flocks: a benchmark study in collective animal behaviour. Anim Behav 76:201-215

Ballerini M, Cabibbo N, Candelier R, Cavagna A, Cisbani E, Giardina I, Lecomte V, Orlandi A, Parisi G, Procaccini A, Viale M, Zdravkovic $\mathrm{V}$ (2008b) Interaction ruling animal collective behaviour depends on topological rather than metric distance: evidence from a field study. Proc Natl Acad Sci U S A 105:1232-1237

Broom M, Ruxton GD (2005) You can run - or you can hide: optimal strategies for cryptic prey against pursuit predators. Behav Ecol 16: 534-554

Buchanan J, Schick C, Brennan L, Herman S (1988) Merlin predation on wintering dunlins: hunting success and dunlin escape tactics. Wilson Bull 100:108-118

Camazine S, Deneubourg J-L, Franks NR, Sneyd J, Theraulaz G, Bonabeau E (2003) Self-organization in biological systems. Princeton University Press, Princeton

Carere C, Montanino S, Moreschini F, Zoratto F, Chiarotti F, Santucci D, Alleva E (2009) Aerial flocking patterns of wintering starlings, Sturnus vulgaris, under different predation risk. Anim Behav 77: 101-107

Carter J, Lyons NJ, Cole HL, Goldson AR (2008) Subtle cues of predation risk: starlings respond to a predator direction of eye gaze. Proc R Soc Lond B 275:1709-1715

Cavagna A, Del Castro L, Giardina I et al (2015) Flocking and turning: a new model for self-organized collective motion. J Stat Phys 158: $601-627$

Feare CJ (1984) The starling. Oxford University Press, Oxford

Gerlotto F, Bertrand S, Bez N, Gutierrez M (2006) Waves of agitation inside anchovy schools observed with multibeam sonar: a way to transmit information in response to predation. ICES J Mar Sci 63 : $1405-1417$

Goodenough AE, Little N, Carpenter WS, Hart AG (2017) Birds of a feather flock together: insights into starling murmuration behaviour revealed using citizen science. PLoS ONE 12:e0179277

Grimm V, Revilla E, Berger U, Jeltsch F, Mooij WM, Railsback SF, Thulke H, Weiner J, Wiegand T, DeAngelis DL (2005) Patternoriented modeling of agent-based complex systems: lessons from ecology. Science 310:987-991

Hemelrijk CK, Hildenbrandt H (2008) Self-organized shape and frontal density of fish schools. Ethology 114:245-254

Hemelrijk CK, Hildenbrandt H (2011) Some causes of the variable shape of flocks of birds. PLoS One 6:e22479 
Hemelrijk CK, Hildenbrandt H (2012) Schools of fish and flocks of birds: their shape and internal structure by self-organization. Interface Focus 2:726-737

Hemelrijk CK, Hildenbrandt H (2015a) Scale-free correlations, influential neighbours and speed control in flocks of birds. J Stat Phys 158: 563-578

Hemelrijk CK, Hildenbrandt H (2015b) Diffusion and topological neighbours in flocks of starlings: relating a model to empirical data. PLoS One 10:e126913

Hemelrijk CK, van Zuidam L, Hildenbrandt H (2015) What underlies waves of agitation in starling flocks. Behav Ecol Sociobiol 69: 755-764

Hildenbrandt H, Carere C, Hemelrijk CK (2010) Self-organized aerial displays of thousands of starlings: a model. Behav Ecol 21:1349 1359

Ouellette NT (2019) Flowing crowds. Science 363:27-28
Potts WK (1984) The chorus-line hypothesis of manoeuvre coordination in avian flocks. Nature 309:344-345

Procaccini A, Orlandi A, Cavagna A, Giardina I, Zoratto F, Santucci D, Chiarotti F, Hemelrijk CK, Alleva E, Parisi G, Carere C (2011) Propagating waves in starling, Sturnus vulgaris, flocks under predation. Anim Behav 82:759-765

Storms RF, Carere C, Zoratto F, Hemelrijk CK (2019) Complex patterns of collective escape in starling flocks under predation. Behav Ecol Sociobiol 73:10

Sumpter DJT, Mann RP, Perna A (2012) The modelling cycle for collective animal behaviour. Interface Focus 2:764-773

Publisher's note Springer Nature remains neutral with regard to jurisdictional claims in published maps and institutional affiliations. 